\title{
On a Fourth Order Accurate Implicit Finite Difference Scheme for Hyperbolic Conservation Laws: I. Nonstiff Strongly Dynamic Problems
}

\author{
By Amiram Harten* and Hillel Tal-Ezer**
}

\begin{abstract}
An implicit finite difference method of fourth order accuracy (in space and time) is introduced for the numerical solution of one-dimensional systems of hyperbolic conservation laws. The basic form of this method is a straightforward generalization of the Crank-Nicholson scheme: it is a two-level scheme which is unconditionally stable and nondissipative. The scheme is compact, i.e., it uses only 3 mesh points at level $t$ and 3 mesh points at level $t+\Delta t$.

In this paper, the first in a series, we present a dissipative version of the basic method which is conditionally stable under the CFL (Courant-Friedrichs-Lewy) condition. This version is particularly useful for numerical solution of problems with strong but nonstiff dynamic features, where the CFL restriction is reasonable on accuracy grounds.

Numerical results are presented to illustrate properties of the proposed scheme.
\end{abstract}

I. Introduction. We consider here numerical solutions of a one-dimensional system of hyperbolic conservation laws

$$
w_{t}+f(w)_{x}=0 \text {. }
$$

Here $w(x, t)$ is an $m$-vector of unknowns and $f(w)$ is a vector-valued function of $m$ components. The system (1.1) is said to be (strictly) hyperbolic when all eigenvalues $a_{1}(w), \ldots, a_{m}(w)$ of the Jacobian matrix

$$
A(w)=\operatorname{grad}_{w} f
$$

are real and distinct; the eigenvalues $a_{k}(w)$ are also referred to as characteristic speeds. Throughout this paper we denote by $v_{j}^{n}=v(j \Delta x, n \Delta t)$ a discrete approximation to solutions $w(x, t)$ of (1.1), where $\Delta x$ and $\Delta t$ are the space and time increments, respectively.

In this paper we present an implicit finite difference method of fourth order accuracy (in space and time, i.e., a 4-4 scheme) for the solution of (1.1). Such a method involves two subjects of much dispute: explicit methods versus implicit methods and higher order accuracy versus lower order accuracy. We can give

Received June 4, 1979; revised May 5, 1980.

1980 Mathematics Subject Classification. Primary 65M05; Secondary 35L65, 76L05.

Key words and phrases. Implicit finite difference scheme, fourth order accuracy, hyperbolic conservation laws.

* On leave from the Department of Mathematics, Tel-Aviv University, Tel-Aviv, Israel.

**Work done towards partial fulfillment of the requirements of M.Sc. degree in the Department of Mathematics, Tel-Aviv University, Tel-Aviv, Israel.

This report was prepared as a result of work performed under NASA Contract No. NAS1-14101 while the authors were in residence at ICASE, NASA Langley Research Center, Hampton, Virginia 23665. 
arguments in favor of both sides in this controversy (as a choice of "best scheme" is very much problem dependent), and we therefore refrain from making further comments on these issues. We refer the interested reader to the excellent review paper by Morton [13] for a description of recent developments and considerations in choosing a numerical scheme. We would also like to suggest a recent paper by Turkel [15] on evaluation of schemes with second order accuracy in time and fourth order accuracy in space (2-4 schemes), explicit as well as implicit. Beam and Warming [2] discuss pros and cons for using implicit schemes.

In Section 2 we review the explicit fourth order accurate scheme of Abarbanel, Gottlieb, and Turkel [1], and the Crank-Nicholson type implicit schemes of Beam and Warming.

In Section 3 we derive a two-level implicit fourth order accurate method which we consider to be a generalization of the Crank-Nicholson scheme-it is unconditionally stable, nondissipative, and uses the same stencil of mesh points: 3 at level $n$ and 3 at the advanced level $n+1$. Analysis presented in this section, as well as numerical experiments not reported here, indicate that there are cases where the coefficient matrix is not invertible in the classical sense. Further analysis and description of algorithms that overcome this difficulty are deferred to future papers.

In Section 4 we present a version of the basic method which is dissipative and conditionally stable under the CFL (Courant-Friedrichs-Lewy) condition

$$
\frac{\Delta t}{\Delta x} \max _{k, w}\left|a_{k}(w)\right| \leqslant 1 \text {. }
$$

This algorithm is particularly useful for numerical solution of problems with strong but nonstiff dynamic features, i.e., where temporal derivatives are at least as large as spatial derivatives, but all characteristic speeds are of the same magnitude. Under such circumstances the CFL restriction (1.3) is acceptable on accuracy grounds.

In Section 5 we discuss the variable coefficient case and in Section 6 the nonlinear case, where special attention is given to the computation of shock waves.

II. Numerical Background. Abarbanel, Gottlieb, and Turkel [1] have recently developed three different versions of fully fourth order accurate (4-4 accuracy) explicit schemes for the solution of (1.1). Their best scheme is the following two-level, four-step algorithm:

$$
\begin{aligned}
v^{(1)} & =\mu v^{n}-\frac{1}{2} \lambda \delta f^{n}, \\
v^{(2)} & =\frac{1}{2}\left(3-\mu^{2}\right) v^{n}-\frac{1}{2} \lambda \delta f^{(1)}, \\
v^{(3)} & =\frac{1}{2}\left(3-\mu^{2}\right) \mu v^{n}-\lambda \delta\left[f^{(2)}+\frac{1}{2}\left(1-\mu^{2}\right) f^{n}\right], \\
v^{(n+1)} & =v^{n}-\frac{1}{6} \lambda \delta\left[f^{(3)}+2 \mu f^{(2)}+2\left(2-\mu^{2}\right) f^{(1)}+\frac{1}{2}\left(3-\mu^{2}\right) \mu f^{n}\right],
\end{aligned}
$$

where $\mu$ and $\delta$ are the commonly used operators

$$
\mu F_{j}=\frac{1}{2}\left(f_{j+1 / 2}+F_{j-1 / 2}\right), \quad \delta F_{j}=F_{j+1 / 2}-F_{j-1 / 2}
$$

$\lambda=\Delta t / \Delta x$ and $f^{n}=f\left(v^{n}\right), f^{(i)}=f\left(v^{(i)}\right), i=1,2,3$. The explicit scheme (2.1) uses a centered stencil of mesh points: 5 points at level $n$ and 1 point at level $n+1$. The scheme (2.1) is dissipative and stable under the optimal CFL condition (1.3). 
One constructs an implicit Crank-Nicholson type scheme for the solution of (1.1) by replacing the integral in the relation

$$
w^{n+1}=w^{n}+\int_{t}^{t+\Delta t} w_{t} d t
$$

with a trapezoidal rule, i.e.,

$$
w^{n+1}=w^{n}+\frac{\Delta t}{2}\left(w_{t}^{n}+w_{t}^{n+1}\right)+O\left((\Delta t)^{3}\right)
$$

and then substituting (from the partial differential equation (1.1)) $w_{t}=-f_{x}$ to obtain

$$
w^{n+1}+\frac{\Delta t}{2} f_{x}^{n+1}=w^{n}-\frac{\Delta t}{2} f_{x}^{n}+O\left((\Delta t)^{3}\right) .
$$

To introduce spatial discretization, we make use of the following finite difference operators

$$
\begin{gathered}
\frac{1}{\Delta x} \mu \delta f=f_{x}+O\left((\Delta x)^{2}\right), \\
\frac{1}{\Delta x} \frac{\mu \delta}{1+\delta^{2} / 6} f=f_{x}+O\left((\Delta x)^{4}\right) .
\end{gathered}
$$

Approximating $f_{x}$ in (2.4b) by the second order centered difference (2.5a) results in the nonlinear scheme

$$
v^{n+1}+\frac{\lambda}{2} \mu \delta f^{n+1}=v^{n}-\frac{\lambda}{2} \mu \delta f^{n}
$$

which is second order accurate in time and space (2-2 scheme).

Using the fourth order Padé formula (2.5b) to approximate $f_{x}$ in $(2.4 \mathrm{~b})$, one obtains, upon clearing the denominator, the nonlinear scheme

$$
\left(1+\delta^{2} / 6\right) v^{n+1}+\frac{\lambda}{2} \mu \delta f^{n+1}=\left(1+\delta^{2} / 6\right) v^{n}-\frac{\lambda}{2} \mu \delta f^{n}
$$

This scheme is second order accurate in time but fourth order accurate in space (2-4 scheme).

Both schemes (2.6a) and (2.6b) are unconditionally stable, nondissipative and have the same stencil of mesh points: 3 points at level $n$ and 3 points at level $n+1$. Clearly, under most circumstances, it is preferable to use the more accurate $2-4$ scheme (2.6b) rather than the (2-2) scheme (2.6a) (see [15]), as there is a relatively small amount of extra work to be invested.

One may linearize (2.6) by expanding $f^{n+1}$ around $v^{n}$ in the following way

$$
f^{n+1}=f^{n}+A^{n}\left(v^{n+1}-v^{n}\right)+O\left((\Delta t)^{2}\right) .
$$

Denoting $\Delta v^{n}=v^{n+1}-v^{n}$, we rewrite the linearized (2.6) in the computationally convenient $\Delta$-formulation of Beam and Warming [2]; (2.6a) and (2.6b) become, respectively,

$$
\begin{gathered}
\left(1+\frac{\lambda}{2} \mu \delta A^{n}\right) \Delta v^{n}=-\lambda \mu \delta f^{n}, \\
\left(1+\delta^{2} / 6+\frac{\lambda}{2} \mu \delta A^{n}\right) \Delta v^{n}=-\lambda \mu \delta f^{n} .
\end{gathered}
$$

Equations (2.8) constitute a block tridiagonal system of linear algebraic equations. This tridiagonal system of equations is diagonally dominant for CFL numbers 
smaller than 2 in the 2-2 scheme (2.8a) and for CFL numbers smaller than $4 / 3$ in the $2-4$ scheme $(2.8 \mathrm{~b})$; it can then be solved by an inexpensive direct $L U$ factorization (see [9, pp. 55-61]). Analysis of the scalar constant coefficient case and numerical experience indicate that direct factorization without prohibitively large round-off error is possible for larger CFL numbers as well. (This is not the case when parabolic terms are added to (1.1); see [8].)

The linearization of the schemes (2.6) increases memory storage requirement considerably; as a result, extension of these linearized schemes to multi-dimensional problems can be handled only by ADI (alternate direction) factorization or spatial operator splitting. One may attempt to solve the nonlinear scheme (2.6) directly by some iterative method. Gustafsson in [4] used a quasi-Newton iterative algorithm; it seems that this algorithm is always less efficient than linearization. Wirz, De Schutter, and Turi, in a recent paper [16], present a semi-implicit relaxation technique for the solution of the one-dimensional problem (1.1). The nonlinear $2-4$ scheme $(2.6 \mathrm{~b})$ is cleverly written as a system

$$
\begin{gathered}
v_{j}^{n+1}=v_{j}^{n}+\frac{\lambda}{2}\left(P_{j}^{n+1}+P_{j}^{n}\right), \quad n=0, \ldots, \\
\left(1+\delta^{2} / 6\right) P_{j}^{n+1}+\mu \delta f_{j}^{n+1}=0, \quad j=1,2, \ldots, M-1 .
\end{gathered}
$$

Here $P=-f_{x} / \Delta x$. The solution to (2.9) is obtained by explicitly sweeping forward and backward on the bounded spatial direction. This procedure reduces the memory storage requirement at the expense of more flux computations. The merit of using (2.9) instead of linearization has yet to be evaluated.

III. Basic 4-4 Formulation. In this section we derive and analyze a fully fourth order accurate variant of $(2.8 b)$. To obtain fourth order accuracy in time we discretize the integral in (2.3) by a trapezoidal rule with "end correction" (see [3, p. 105])

$$
w^{n+1}=w^{n}+\frac{\Delta t}{2}\left(w_{t}^{n}+w_{t}^{n+1}\right)+\frac{(\Delta t)^{2}}{12}\left(w_{t t}^{n}-w_{t t}^{n+1}\right)+O\left((\Delta t)^{5}\right) .
$$

Next we substitute temporal derivatives into (3.1) by spatial derivatives via the differential equation (1.1), i.e., $w_{t}=-f_{x}, w_{t t}=-f_{t x}=-\left(A w_{t}\right)_{x}=\left(A f_{x}\right)_{x}$, to get

$$
\begin{aligned}
w^{n+1}+\frac{\Delta t}{2} & f_{x}^{n+1}+\frac{(\Delta t)^{2}}{12}\left(A^{n+1} f_{x}^{n+1}\right)_{x} \\
= & w^{n}-\frac{\Delta t}{2} f_{x}^{n}+\frac{(\Delta t)^{2}}{12}\left(A^{n} f_{x}^{n}\right)_{x}+O\left((\Delta t)^{5}\right) .
\end{aligned}
$$

To obtain fourth order accuracy in space, we approximate $f_{x}$ in the term $\Delta t f_{x} / 2$ in (3.2) by the fourth order accurate Padé formula (2.5b), and we approximate the second order derivative $\left(A f_{x}\right)_{x}$ by

$$
\left(A f_{x}\right)_{x}=\frac{1}{(\Delta x)^{2}} \delta A \delta f+O\left((\Delta x)^{2}\right) .
$$

The last approximation is sufficiently accurate since the term $\left(A f_{x}\right)_{x}$ is multiplied by $(\Delta t)^{2}$, and it appears in (3.2) in a form of temporal difference, $\left.\left(A f_{x}\right)_{x}\right|_{n} ^{n+1}$, which contributes another order in $\Delta t$. Upon clearing the denominator $\left(1+\delta^{2} / 6\right)$ in the 
Padé approximation (2.5b) and neglecting the terms

$$
\left.\delta^{2} \frac{(\Delta t)^{2}}{(\Delta x)^{2}} \delta A \delta f\right|_{n} ^{n+1}=O\left((\Delta t)^{3}(\Delta x)^{2}\right)
$$

as being of the same order as the truncation error, we get the 4-4 scheme

$$
\begin{aligned}
\left(1+\delta^{2} / 6\right) v^{n+1} & +\frac{\lambda}{2} \mu \delta f^{n+1}+\frac{\lambda^{2}}{12} \delta A^{n+1} \delta f^{n+1} \\
& =\left(1+\delta^{2} / 6\right) v^{n}-\frac{\lambda}{2} \mu \delta f^{n}+\frac{\lambda^{2}}{12} \delta A^{n} \delta f^{n}
\end{aligned}
$$

Comparing the 4-4 scheme (3.3) with the 2-2 scheme (2.6a) and the 2-4 scheme (2.6b), we see that (3.3) can be regarded as a generalization of the other two. Since the 4-4 scheme uses the same stencil of mesh points as the 2-2 scheme and the 2-4 scheme, and since this is the most compact stencil to achieve a 4-4 accuracy, we feel that referring to (3.3) as a compact scheme is justified.

To linearize the scheme (3.3) and still preserve the temporal fourth order accuracy, we first obtain a second order accurate approximation $\hat{v}$ to the solution of (1.1) at level $n+1$

$$
\hat{v}=v^{n+1}+O\left((\Delta t)^{3}\right) .
$$

Then we expand $f^{n+1}$ and $A^{n+1}$ around $\hat{v}$ in the following way

$$
\begin{gathered}
f^{n+1}=\hat{f}+\hat{A}\left(v^{n+1}-\hat{v}\right)+O\left(\left\|v^{n+1}-\hat{v}\right\|^{2}\right) \equiv \tilde{f}+\hat{A} \Delta v^{n}+O\left((\Delta t)^{6}\right) \\
A^{n+1}=\hat{A}+O\left((\Delta t)^{3}\right)
\end{gathered}
$$

where $\hat{A}=A(\hat{v}), \hat{f}=f(\hat{v})$, and $\tilde{f}$ denotes $\tilde{f}=\hat{f}+\hat{A}\left(v^{n}-\hat{v}\right)$ and as before $\Delta v^{n}=$ $v^{n+1}-v^{n}$. Using the linearization (3.4) in the scheme (3.3), we get the following $\Delta$-form

(3.5) $\left(1+\delta^{2} / 6+\frac{\lambda}{2} \mu \delta \hat{A}+\frac{\lambda^{2}}{12} \delta \hat{A} \delta \hat{A}\right) \Delta v^{n}=-\frac{\lambda}{2} \mu \delta\left(f^{n}+\hat{f}\right)+\frac{\lambda^{2}}{12} \delta\left(A^{n} \delta f^{n}-\hat{A} \delta \hat{f}\right)$.

We remark that one can also express $w_{t t}$ in (3.1) as $w_{t t}=\left(A^{2} w_{x}\right)_{x}$; this leads to a slightly different form of (3.5).

Next we analyze the 4-4 scheme in the constant coefficient case

$$
w_{t}+A w_{x}=0, \quad A=\text { constant matrix. }
$$

In this case $f(w)=A w$, therefore in (3.5) $f^{n}=\tilde{f} \equiv A v^{n}$ and $A^{n}=\hat{A} \equiv A$. Thus, for any method of computing $\hat{v}$ in (3.4a), the linearized scheme (3.5) becomes the same as (3.3) with $f=A v$, i.e.,

(3.6) $\left(1+\delta^{2} / 6+\frac{\lambda}{2} A \mu \delta+\frac{\lambda^{2}}{12} A^{2} \delta^{2}\right) v^{n+1}=\left(1+\delta^{2} / 6-\frac{\lambda}{2} A \mu \delta+\frac{\lambda^{2}}{12} A^{2} \delta^{2}\right) v^{n}$.

We perform a Fourier analysis of the scalar case by substituting in (3.6)

$$
v_{j}^{l}=V^{l} e^{i k j \Delta x} \equiv V^{l} e^{i j \xi}, \quad \xi=k \Delta x, l=n, n+1 .
$$

Let $\nu=a \Delta t / \Delta x$ denote the CFL number. The amplification factor $g(\xi, \nu)$, defined 
by $v^{n+1}=g(\xi, \nu) v^{n}$, is

$$
g(\xi, \nu)=\frac{1-\frac{1}{3}\left(2+\nu^{2}\right) \sin ^{2} \frac{\xi}{2}-i \frac{\nu}{2} \sin \xi}{1-\frac{1}{3}\left(2+\nu^{2}\right) \sin ^{2} \frac{\xi}{2}+i \frac{\nu}{2} \sin \xi} .
$$

Thus, $g(\xi, \nu)$ is the ratio of a number and its complex conjugate, and therefore

$$
|g(\xi, \nu)|=1, \quad g(\xi, \nu)=e^{-i \varphi(\xi, \nu)}
$$

where $\varphi(\xi, \nu)$, the phase shift of the scheme per time step, is the following real number

$$
\varphi(\xi, \nu)=2 \operatorname{tg}^{-1}\left[\frac{\frac{1}{2} \nu \sin \xi}{1-\frac{1}{3}\left(2+\nu^{2}\right) \sin ^{2} \frac{\xi}{2}}\right] .
$$

Just as for the 2-2 and the 2-4 schemes in (2.6), the 4-4 scheme is unconditionally stable, nondissipative, and its only error is a phase error. We point out again that the linear stability of (3.3) does not depend on the way $\hat{v}$ in (3.4a) is calculated.

The finite difference operator on the LHS of (3.5) and (3.6), $L_{l}$, can be rewritten in the constant coefficient case as

$$
\begin{aligned}
\left(L_{l} v\right)_{j} \equiv & \frac{1}{12}(\nu-1)(\nu-2) v_{j-1}-\frac{1}{6}(\nu-2)(\nu+2) v_{j} \\
& +\frac{1}{12}(\nu+1)(\nu+2) v_{j+1} .
\end{aligned}
$$

This finite difference operator gives rise to a tridiagonal coefficient matrix. It is easy to see that this matrix is diagonally dominant, and consequently nonsingular, if $|\nu|<1$. When diagonally dominant, the system of linear equation (3.5) can be safely solved by a direct $L U$ factorization, since in this case the accumulation of round-off errors is not prohibitively large (see [9, pp. 55-61]). We remind the reader that the tridiagonal matrix coefficient of the $2-2$ scheme (2.8a) is diagonally dominant when $|\nu|<2$, and that of the 2-4 scheme $(2.8 \mathrm{~b})$ is diagonally dominant when $|\nu|<4 / 3$. It can be shown that even when diagonal dominance is lost, the coefficient matrices of the 2-2 and the 2-4 schemes remain nonsingular and that direct $L U$ factorization is still possible. Unfortunately, this is not so for the 4-4 scheme (3.5).

The finite difference operator $L_{l}$ in (3.8) becomes singular when $|\nu|=1$; this is easily seen from the fact that its Fourier symbol vanishes for $|\nu|=1$ and $\xi=\pi$. Indeed, the mesh oscillation function $v_{j}=e^{i j \pi}=(-1)^{j}$ is an eigenfunction of $L_{l}$ with a zero eigenvalue. Consequently applying the 4-4 scheme (3.5) to problems with $|\nu|>1$ may result in an ill-conditioned coefficient matrix, or even a singular one. This difficulty is not inherent in the method as indicated by the regularity of the amplification factor (3.7a) and the phase shift (3.7c) for all values of the CFL number. A more detailed analysis will be given in future papers; there we shall modify the 4-4 method so as to be applicable to problems which involve large CFL numbers, such as problems with parasitic stiffness (i.e. waves with negligible amplitude but large characteristic speed) and problems which involve marching to a steady state. 
In the present paper, we consider possible applications to nonstiff problems with strongly dynamic features, i.e., where temporal derivatives are at least as large as spatial derivatives, but all waves have speeds of propagation of the same magnitude and have to be resolved equally well. In the next section, we shall present an inherently dissipative variant of (3.3) which is more robust. This scheme is conditionally stable under the CFL restriction (1.3), but for the type of problems considered here this is not overly restrictive since it is in any case needed by accuracy considerations.

IV. Dissipative 4-4. Let $L_{2}$ be a second order accurate finite difference approximation to the solution operator of (1.1)

$$
\hat{v}=L_{2} v^{n}, \quad \hat{v}=w^{n+1}+O\left((\Delta t)^{3}\right) .
$$

Assuming $f(w)$ and $A(w)$ to be sufficiently smooth functions, we have from (4.1)

$$
\hat{f} \equiv f(\hat{v})=f\left(w^{n+1}\right)+O\left((\Delta t)^{3}\right), \quad \hat{A} \equiv A(\hat{v})=A\left(w^{n+1}\right)+O\left((\Delta t)^{3}\right)
$$

Thus,

$$
\delta \hat{A} \delta \hat{f}=\delta A^{n+1} \delta f^{n+1}+O\left((\Delta t)^{3}(\Delta x)^{2}\right) .
$$

Therefore we can replace $\delta A^{n+1} \delta f^{n+1}$ in (3.3) by $\delta \hat{A} \delta \hat{f}$ without disrupting the fourth order accuracy of the scheme. Linearizing $f^{n+1}$ in (3.3) by (3.4b) and using (4.2b), we get

$$
\left(1+\delta^{2} / 6+\frac{\lambda}{2} \mu \delta \hat{A}\right) \Delta v^{n}=-\frac{\lambda}{2} \mu \delta\left(f^{n}+\hat{f}\right)+\frac{\lambda^{2}}{12} \delta\left(A^{n} \delta f^{n}-\hat{A} \delta \hat{f}\right),
$$

where as before $\Delta v^{n}=v^{n+1}-v^{n}, \tilde{f}=\hat{f}+\hat{A}\left(v^{n}-\hat{v}\right)$. We observe that the coefficient matrix in (4.3) is identical in structure to that of the $2-4$ scheme (2.6b); this matrix is diagonally dominant for CFL numbers smaller than $4 / 3$.

Next, we study the linear stability of (4.4) by Fourier analysis. In the constant coefficient case $f(w)=A w, A=$ const, we have $\hat{A}=A^{n} \equiv A, f^{n}=\tilde{f} \equiv A v^{n}$, and (4.3) becomes

$$
\left(1-\delta^{2} / 6+\frac{\nu}{2} \mu \delta\right) \Delta v^{n}=-\nu \mu \delta v^{n}+\frac{\nu^{2}}{12} \delta^{2}\left(v^{n}-\hat{v}\right),
$$

where $\nu$ is the CFL number. Substituting $v_{j}^{l}=V^{l} e^{i j k \Delta x} \equiv V^{l} e^{i j \xi}, \hat{v}_{j}=\hat{V} e^{i j \xi}, \hat{V}=$ $g_{2}(\xi, v) V^{n}$ into (4.4), we obtain the following amplification factor

$$
g(\xi, \nu)=1-\frac{i \nu \sin \xi+\frac{1}{3} \nu^{2} \sin ^{2} \frac{\xi}{2}\left(1-g_{2}(\xi, \nu)\right)}{1-\frac{2}{3} \sin ^{2} \frac{\xi}{2}+i \frac{\xi}{2} \sin \xi} .
$$

Here $g_{2}(\xi, \nu)$ is the amplification factor of $L_{2}$ in (4.1).

We take $L_{2}$ in (4.1) to be the explicit second order accurate Lax-Wendroff (LW) scheme (see [11])

$$
\hat{v}=v^{n}-\lambda A \mu \delta v^{n}+\frac{\lambda^{2}}{2} A^{2} \delta^{2} v^{n} .
$$

The amplification factor of this scheme is

$$
g_{2}(\xi, \nu)=1-2 \nu^{2} y-i \nu \sin \xi .
$$


The amplitude of the amplification factor (4.5) with (4.7) is

$$
|g(\xi, \nu)|^{2}=1-4 \nu^{4}\left(1-\nu^{2}\right) y^{3} \frac{1+\nu^{2} y}{(3-2 y)^{2}+9 \nu^{2} y(1-y)},
$$

where $y=\sin ^{2} \xi / 2$. Thus, the scheme (4.4), (4.6) is stable for $|\nu|<1$. For small $\xi$, we get

$$
|g(\xi, \nu)|=1-\frac{1}{288} \nu^{4}\left(1-\nu^{2}\right) \xi^{6}+O\left(\xi^{7}\right),
$$

which shows that the scheme is dissipative of order 6 . We note that the potentially harmful mesh oscillation mode $v_{j}=(-1)^{j}$, corresponding to $\xi=\pi$, is being damped by (4.4), for $0<|\nu|<1$,

$$
|g(\pi, \nu)|^{2}=1-4 \nu^{4}\left(1-\nu^{4}\right) .
$$

This mode is completely annihilated for $|\nu|=2^{-1 / 4} \approx 0.84$. The dissipation in the 4-4 scheme (4.4) is generated by the dissipation in the LW scheme in (4.6), for which

$$
\left|g_{2}(\xi, \nu)\right|^{2}=1-4 \nu^{2}\left(1-\nu^{2}\right) y^{2} .
$$

Comparing (4.9) with (4.8), we see that the basic properties of the $\mathrm{LW}$ dissipation are inherited by the $4-4$ scheme.
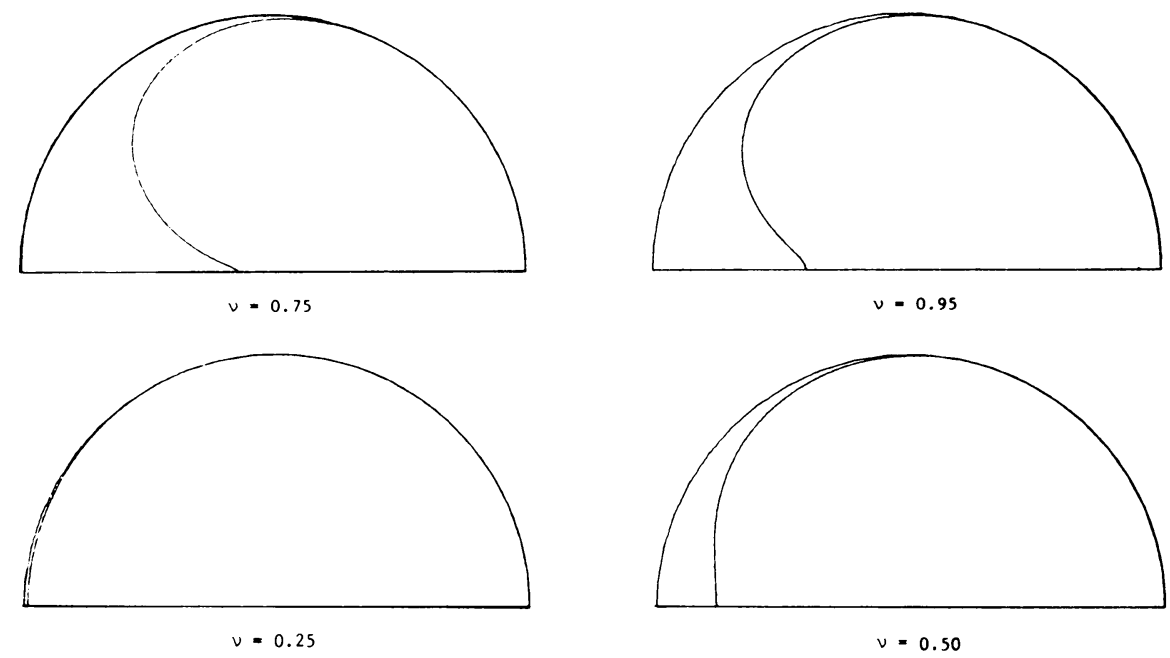

Figure 1

Amplification factor (4.8a) of the dissipative 4-4 scheme

In Figure 1, we illustrate the nature of the dissipation in the 4-4 scheme (4.4) by polar plots of $|g(\xi, \nu)|, 0<\xi<\pi$, for $\nu=0.25,0.50,0.75,0.95$. The distance between the curve of $|g(\xi, \nu)|$ and the unit semicircle measures the amount of dissipation in the scheme. Just as for the LW scheme (4.9), the dissipation in the 4-4 scheme (4.8) vanishes for $\nu=0,|\nu|=1$; therefore one should use a CFL number smaller than 1. From Figure 1 we see that a choice of $\nu=0.95$ is a good one: it is very accurate for the long waves (see (4.8b)) but damps considerably the harmful short waves (see (4.8c)). The vanishing of the dissipation for $\nu=0$ can cause nonlinear instabilities for the 4-4 scheme (4.4) as well as for the LW scheme; we shall discuss this difficulty in Section 6. 
To study the phase error in the 4-4 scheme (4.4) we rewrite the amplification factor as

$$
g(\xi, \nu) \equiv|g(\xi, \nu)| e^{-i \varphi(\xi, \nu)},
$$

where $\varphi(\xi, \nu)$ is a real number which describes the phase shift per time-step assigned by the schemes to a sinusoidal wave with wave-number $\xi$, and is given by

$$
\varphi(\xi, \nu)=\operatorname{tg}^{-1}\left[\nu \sin \xi \frac{-\nu^{2}\left(2+3 \nu^{2}\right) y^{2}+3\left(\nu^{2}-2\right) y+9}{10 \nu^{4} y^{3}+\left(9 \nu^{2}-12 \nu^{4}+4\right) y^{2}-3\left(3 \nu^{2}+4\right) y+9}\right]
$$

Following [2], we illustrate the nature of the phase error in the 4-4 scheme by polar plots of $\varphi(\xi, \nu) / \varphi_{E}, 0<\xi<\pi$, where $\varphi_{E}=a k \Delta t=\nu \xi$ is the phase shift per time-step in the exact spatially periodic solution of the scalar wave equation $w_{t}=a w_{x}=0$. In Figure 2 we compare the phase error of the dissipative 4-4 scheme (4.10) with those of the nondissipative 4-4 (3.7c) and the 2-4 scheme (2.8b) for $\nu=0.25,0.50,0.75,0.95$. The deviation of the curves $\varphi / \varphi_{E}$ from the unit semicircle measures the relative phase error; $\varphi / \varphi_{E}<1$ signifies lagging phase error. Similar polar plots for the 2-2 scheme (2.8a) are available in [2].
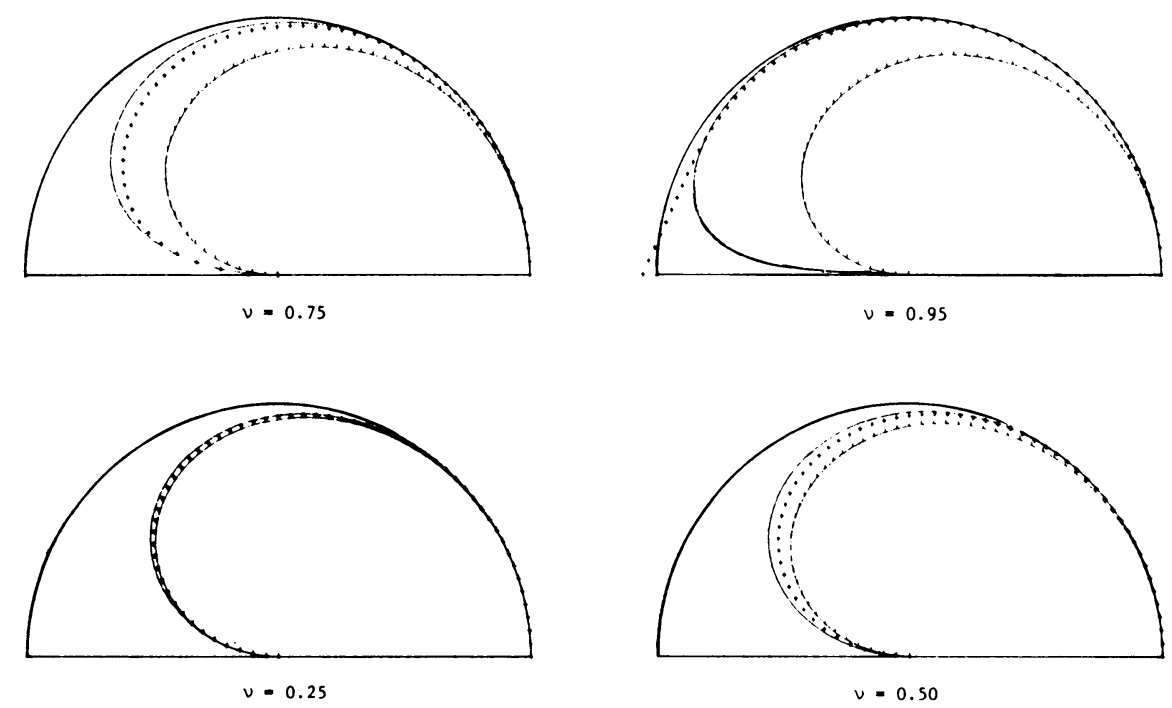

Figure 2

Relative phase error $\varphi / \varphi_{E} ; \quad-$ nondissipative 4-4, +++ dissipative 4-4, $++1+2-4$.

We make the following observations about Figure 2:

(1) The accuracy in phase of the 4-4 schemes, dissipative as well as nondissipative, improves as the CFL number increases. In fact $\varphi=\varphi_{E}$ for $\nu=1$.

(2) The accuracy of the nondissipative 4-4 is slightly better than that of the dissipative 4-4, but both are of the same quality.

(3) The accuracy of the 2-4 scheme is about the same as that of the $4-4$ schemes for $\nu<0.25$, but its accuracy deteriorates rapidly with increasing CFL number. The last statement was verified experimentally in more practical problems by Turkel in [15]. 
From a combined study of amplitude and phase error, we conclude that the best choice of a CFL number for the dissipative 4-4 is in a neighborhood of $\nu=0.95$.

V. Variable Coefficient Problems. In this section, we consider a hyperbolic variable coefficient problem of the form

$$
w_{t}+A(x) w_{x}=0 .
$$

In this case, $w_{t}=-A(x) w_{x}, w_{t t}=A(x)\left[A(x) w_{x}\right]_{x}$. To obtain fourth order accuracy in the spatial discretization, we approximate the term $A(x) w_{x}$ by a modified Padé formula

$$
A(x) w_{x}=\frac{1}{3 \Delta x} \frac{4 \mu A \delta-A \mu \delta}{1+\delta^{2} / 6} w+O\left((\Delta x)^{4}\right),
$$

and, just as before, it is sufficient to approximate the higher order term $A(x)\left[A(x) w_{x}\right]_{x}$ by

$$
A(x)\left[A(x) w_{x}\right]_{x}=\frac{1}{(\Delta x)^{2}} A \delta A \delta w+O\left((\Delta x)^{2}\right) .
$$

Rearranging terms, we get the following expression with $\alpha_{24}=\alpha_{44}=1$ for the nondissipative 4-4 scheme

$$
\begin{gathered}
{\left[1+\alpha_{2-4} \delta^{2} / 6+\frac{\lambda}{6}(4 \mu A-A \mu) \delta+\alpha_{44} \frac{\lambda^{2}}{12} A \delta A \delta\right] \Delta v^{n}} \\
=-\frac{\lambda}{3}(4 \mu A-A \mu) \delta v^{n} .
\end{gathered}
$$

The variable coefficient version of the $2-4$ scheme (2.6b) is given by (5.4) with $\alpha_{2-4}=1, \alpha_{4-4}=0$. To simplify our programming, we considered (5.4) with $\alpha_{2-4}=$ $\alpha_{44}=0$ to be the variable coefficient version of the 2-2 scheme (2.6a). In general, it would be simpler to replace the operator $\frac{1}{3}(4 \mu A-A \mu) \delta$ in (5.4) by $A \mu \delta$ to approximate $A \partial / \partial x$ to second order accuracy.

Proceeding as before, we obtain the following algorithm for the dissipative 4-4 scheme (4.3) in the variable coefficient case

$$
\begin{gathered}
\hat{v}=v^{n}-\frac{\lambda}{2} A \mu \delta v^{n}+\frac{\lambda^{2}}{2} A \delta A \delta v^{n} \\
{\left[1+\delta^{2} / 6+\frac{\lambda}{6}(4 \mu A-A \mu) \delta\right] \Delta v^{n}} \\
=-\frac{\lambda}{3}(4 \mu A-A \mu) \delta v^{n}+\frac{\lambda^{2}}{12} A \delta A \delta\left(v^{n}-\hat{v}\right) .
\end{gathered}
$$

Comparing (5.5) with (5.4), we find that the explicit correction in the dissipative 4-4 scheme causes an enlargement of the stencil of mesh points: the number of mesh points at the level $n+1$ remains 3 , but at the "explicit level" $n$ it increases from 3 to 5. However, the computational effort required in the dissipative 4-4 scheme is only slightly larger than that in the nondissipative 4-4 scheme, as all the terms in the RHS of (5.5a) must in any case be computed to set up (5.5b). By way of justification, we remark that adding an explicit dissipation term to the RHS of the nondissipative 4-4 scheme involves 7 mesh points at level $n$. 
Next, we describe some numerical experiments with the periodic, scalar variable coefficient problem ${ }^{* * *}$

$$
w_{t}+\frac{1}{2+\cos x} w_{x}=0
$$

with the initial data

$$
w(x, 0)=2+\sin (2 x+\sin x), \quad-\pi<x<\pi,
$$

and the periodic boundary conditions

$$
w(-\pi, t)=w(\pi, t), \quad t \geqslant 0 .
$$

The exact solution to the problem (5.6a) $-(5.6 \mathrm{c})$ is given by

\begin{tabular}{|c|c|c|c|c|c|c|c|c|}
\hline$n ; M$ & $2-2$ & RATIO & $2-4$ & RATIO & $\begin{array}{l}\text { DISSIPATIVE } \\
4-4 \quad(5.5)\end{array}$ & RATIO & $\begin{array}{l}\text { NONDISSIPATIVE } \\
4-4 \quad(5.4)\end{array}$ & RATIO \\
\hline $20 ; 19$ & $2.290 \times 10^{-1}$ & ----- & $2.370 \times 10^{-2}$ & ---- & $8.188 \times 10^{-3}$ & $-\cdots$ & $5.978 \times 10^{-3}$ & -- \\
\hline $40 ; 39$ & $5.674 \times 10^{-2}$ & ----- & $4.428 \times 10^{-3}$ & ---- & $4.075 \times 10^{-4}$ & $-\cdots-$ & $2.863 \times 10^{-4}$ & --- \\
\hline $80 ; 79$ & $1.374 \times 10^{-2}$ & $\cdots$ & $1.011 \times 10^{-3}$ & --- & $2.337 \times 10^{-5}$ & ---- & $1.628 \times 10^{-5}$ & -- \\
\hline $160 ; 159$ & $3.369 \times 10^{-3}$ & ----- & $2.446 \times 10^{-4}$ & ---- & $1.407 \times 10^{-6}$ & ----- & $9.774 \times 10^{-7}$ & ---- \\
\hline
\end{tabular}

$$
w(x, t)=2+\sin (2 x+\sin x-t) .
$$

\begin{tabular}{|c|c|c|c|c|}
\hline & $2-2$ & $2-4$ & $\begin{array}{l}\text { Dissipative } \\
4-4 \quad(5.5)\end{array}$ & $\begin{array}{l}\text { Nondissipative } \\
4-4 \quad(5.4)\end{array}$ \\
\hline 50 & $7.343 \times 10^{-2}$ & $5.673 \times 10^{-3}$ & $5.002 \times 10^{-4}$ & $3.561 \times 10^{-4}$ \\
\hline 100 & $1.343 \times 10^{-1}$ & $1.121 \times 10^{-2}$ & $7.867 \times 10^{-4}$ & $5.787 \times 10^{-4}$ \\
\hline 150 & $1.899 \times 10^{-1}$ & $1.547 \times 10^{-2}$ & $1.142 \times 10^{-3}$ & $8.373 \times 10^{-4}$ \\
\hline 200 & $2.510 \times 10^{-1}$ & $2.152 \times 10^{-2}$ & $1.554 \times 10^{-3}$ & $1.143 \times 10^{-3}$ \\
\hline 250 & $3.393 \times 10^{-1}$ & $2.836 \times 10^{-2}$ & $2.020 \times 10^{-3}$ & $1.489 \times 10^{-3}$ \\
\hline 300 & $3.820 \times 10^{-1}$ & $3.397 \times 10^{-2}$ & $2.482 \times 10^{-3}$ & $1.827 \times 10^{-3}$ \\
\hline $\begin{array}{l}\text { Average } \\
\text { Accumulation } \\
\text { Per Time Step }\end{array}$ & $1.273 \times 10^{-3}$ & $1.132 \times 10^{-4}$ & $8.273 \times 10^{-6}$ & $6.090 \times 10^{-6}$ \\
\hline
\end{tabular}

Mesh refinement chart-variable coefficient problem (5.6)

TABLE II

Error accumulation-variable coefficient problem (5.6) 
In Table I, we present a mesh refinement chart for the 2-2, 2-4, dissipative 4-4, and the nondissipative 4-4 schemes of this section. Table I shows a sequence of calculations with a constant CFL number $\nu$

$$
\nu=\frac{\Delta t}{\Delta x} \max _{-\pi<x<\pi}|a(x)|=\frac{\Delta t}{\Delta x} \cdot 1=0.95 ; \quad \Delta x=2 \pi / M,
$$

where the number of grid points is successively refined by a factor of 2 . The relative $L_{2}$-errors in Table I are computed at roughly the same physical time.

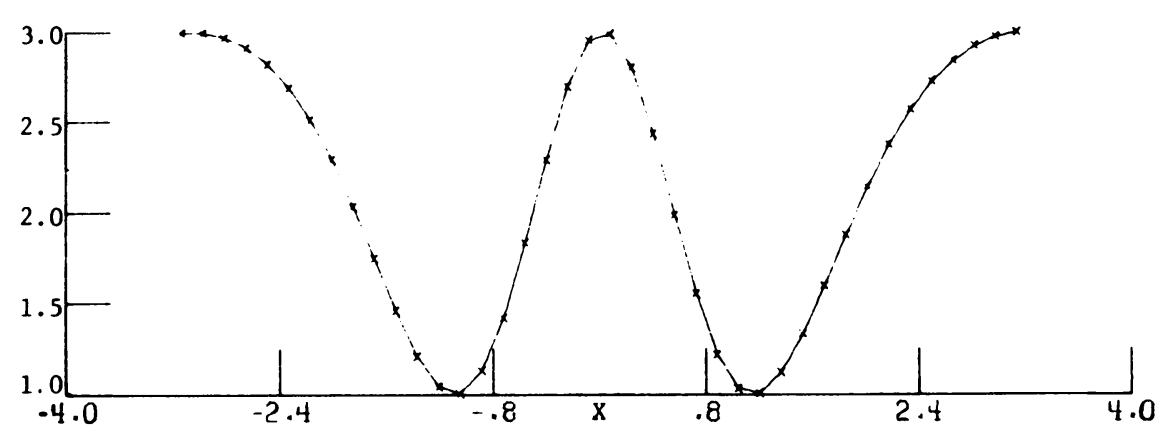

(a) Dissipative 4-4

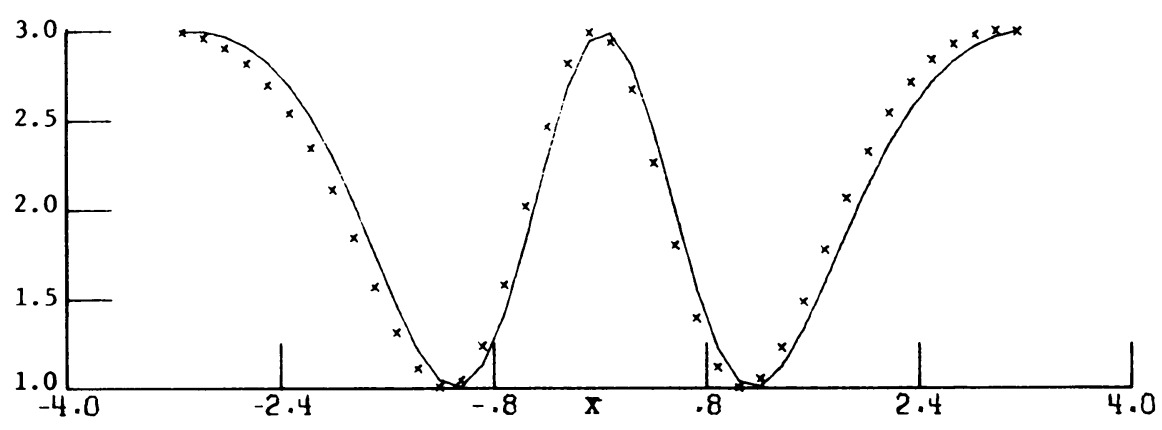

(b) Implic1t 2-4

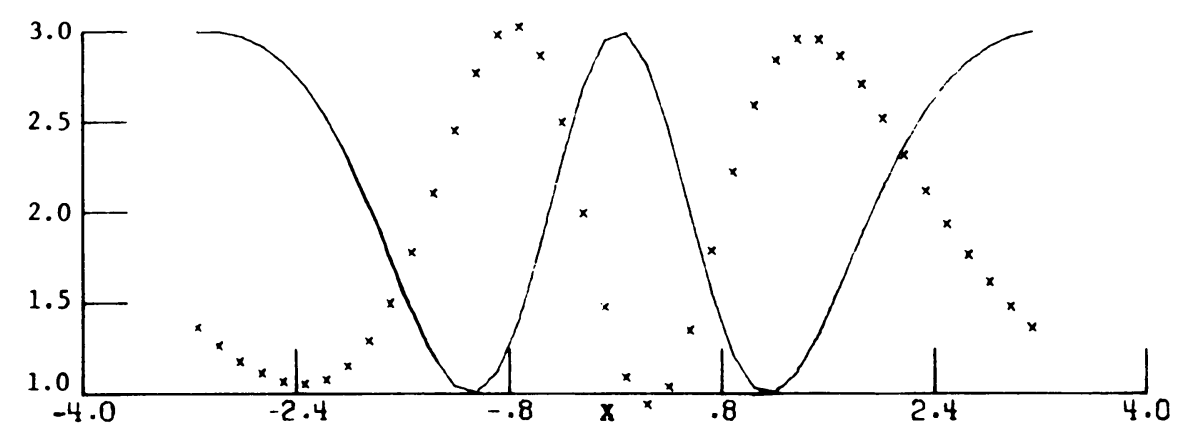

(c) Inplic1t 2-2

Figure 3

Solutions to the variable coefficient problem $(5.6)$ at $t=92.76(n=600)$ 
In Table II, we present error-accumulation data for the schemes in Table I. Table II shows the time history of the relative $L_{2}$-error in a fixed mesh computation for $n=50,100,150,200,250,300 ; \Delta t$ and $\Delta x$ in this calculation are given by (5.7) with $M=39$. In Figure 3, we plot the numerical solutions corresponding to $n=600(t=92.764)$ in Table II and compare them with the exact solution (5.6d). The exact solution is periodic in time with a period of $2 \pi$, thus the results shown in Figure 3 correspond to $92.76 / 2 \pi \approx 14.8$ periods of time. The relative $L_{2}$-error used in Tables I and II is defined by

$$
\left[\frac{\Delta x \Sigma\left(v_{j}-w_{j}\right)^{2}}{\Delta x \Sigma w_{j}^{2}}\right]^{1 / 2},
$$

where $v_{j}$ and $w_{j}$ are the numerical and exact solutions, respectively.

We make the following observations regarding the data of the numerical experiments in Tables I and II and Figure 3:

(1) Table I demonstrates that both the dissipative 4-4 (5.5) and the nondissipative 4-4 ((5.4) with $\left.\alpha_{2-4}=\alpha_{4-4}=1\right)$ schemes are fully fourth order accurate; a refinement by a factor of 2 reduces the error by a factor of approximately $16=2^{4}$. Both schemes have the same quality of results, but the nondissipative 4-4 scheme is consistently better than the dissipative 4-4 scheme.

(2) The 2-2 implicit scheme ((5.4) with $\alpha_{2-4}=\alpha_{4-4}=0$ ) has very poor accuracy even for relatively fine meshes ( $\operatorname{such}$ as 40 points for $\sin (2 x+\sin x),-\pi<x<\pi$ ).

(3) The 2-4 scheme is about 10 times more accurate than the 2-2 scheme, but is much less accurate than the 4-4 schemes. To obtain an error of the order $10^{-4}$ in Table I, the 2-4 scheme uses $M=159, n=160$, while the 4-4 scheme achieves this accuracy with $M=39, n=40$. In this particular case, the 4-4 schemes are roughly 12-14 times more computationally efficient than the 2-4 scheme.

(4) Table II shows a linear accumulation of error for all schemes considered, thus indicating that even the nondissipative schemes are perfectly stable for this variable coefficient problem. In our opinion, the results in this table clearly demonstrate the usefulness of high order accuracy in long term fixed-mesh computations.

VI. Nonlinear Problems. In this section we examine numerical solutions to nonlinear hyperbolic conservation laws (1.1) in both the scalar and the vector case. We consider the case where the 4-4 schemes are linearized around a solution of the one-step Lax-Wendroff scheme. In this case the nondissipative 4-4 scheme (3.5) takes the form

$$
\begin{aligned}
\hat{v} & =v^{n}-\frac{\lambda}{2} \mu \delta f^{n}+\frac{\lambda^{2}}{2} \delta A^{n} \delta f^{n} \\
\left(1+\delta^{2} / 6+\frac{\lambda}{2} \mu \delta \hat{A}+\frac{\lambda^{2}}{12} \delta \hat{A} \delta \hat{A}\right) \Delta v^{n} & \\
& =-\frac{\lambda}{2} \mu \delta\left(f^{n}+\hat{f}\right)+\frac{\lambda^{2}}{12} \delta\left(A^{n} \delta f^{n}-\hat{A} \delta \hat{f}\right)
\end{aligned}
$$

Similarly, the dissipative 4-4 scheme (4.3) with (4.1) takes the form

$$
\hat{v}=v^{n}-\frac{\lambda}{2} \mu \delta f^{n}+\frac{\lambda^{2}}{2} \delta A^{n} \delta f^{n}
$$




$$
\left(1+\delta^{2} / 6+\frac{\lambda}{2} \mu \delta \hat{A}\right) \Delta v^{n}=-\frac{\lambda}{2} \mu \delta\left(f^{n}+\tilde{f}\right)+\frac{\lambda^{2}}{12} \delta\left(A^{n} \delta f^{n}-\hat{A} \delta \hat{f}\right)
$$

As before, $\tilde{f}=\hat{f}+\hat{A}\left(v^{n}-\hat{v}\right), \hat{f}=f(\hat{v}), \hat{A}=A(\hat{v})$.

The choice of the one-step Lax-Wendroff scheme in (6.1) and (6.2) is the most natural one since its calculation involves terms which appear on the right-hand side of Eqs. (6.1b) and (6.2b). This version of the 4-4 schemes uses two flux vector evaluations $\left(f^{n}\right.$ and $\left.\hat{f}\right)$ and two matrix function evaluations $\left(A^{n}\right.$ and $\left.\hat{A}\right)$. More efficient algorithms for (6.1) and (6.2), which use only one matrix and one flux vector evaluations, are possible (see Appendix A in [17]).

The nonlinear case differs from the variable coefficient case in that both the dissipative 4-4 (6.2) and the nondissipative 4-4 (6.1) schemes have the same enlarged stencil of mesh points: 3 points at level $n+1$ and 5 points at the "explicit" level $n$. Since the enlargement of the stencil in the nondissipative 4-4 scheme is due to linearization and not to variation in space (as is evident upon comparing (6.1) with (5.4) and (3.3)), we expect it not to create major difficulties in practical applications of the scheme.

We observe that both 4-4 schemes are in conservation form. This is self-evident from the fact that all terms except $\Delta v^{n}$ in (6.1b) and (6.2b) have a $\delta$ operator in front of them (note that $\mu \delta=\delta \mu$ ). This conservation form is of particular significance for the computation of shock waves, since it guarantees a correct propagation of shock fronts; see [11].

Next, we describe some numerical experiments with the periodic nonlinear scalar problem

$$
w_{t}+w w_{x}=0, \quad-\pi<x<\pi, t>0,
$$

with the initial data

$$
w(x, 0)=d+\sin x ; d=\text { const } ; \quad-\pi<x<\pi,
$$

and the periodic boundary condition

$$
w(-\pi, t)=w(\pi, t), \quad t \geqslant 0 .
$$

It is easy to see that for all values of $d$ the solution to (6.3) is smooth up to $t=1$, at which time a shock wave is formed. After formation this shock decays at a fast rate which is typical to periodic problems. (See Appendix B in [17] for a more detailed description of the solution to (6.3) and the semianalytic algorithm by which we compute it.)

We first discuss the case $d=0$ in (6.3b). Here, a stationary shock develops at $t=1$ at the endpoints $x=\pi$ and $x=-\pi$. Numerical experiments with the 2-2 scheme (2.8a), the 2-4 scheme (2.8b) and the 4-4 schemes (6.1) and (6.2) show that in this case all schemes develop strong nonlinear instabilities after the formation of the shock. This nonlinear instability at stagnation points is well known for Lax-Wendroff type schemes, and is related to their lack of dissipation near zero eigenvalues; see [7], [12] and the references cited there. The occurrence of such nonlinear instabilities can be prevented by supplementing the scheme with a nonlinear dissipation term which does not vanish at stagnation points; see [12] and [6]. 
TABLE III

Mesh refinement chart-nonlinear problem (6.3)

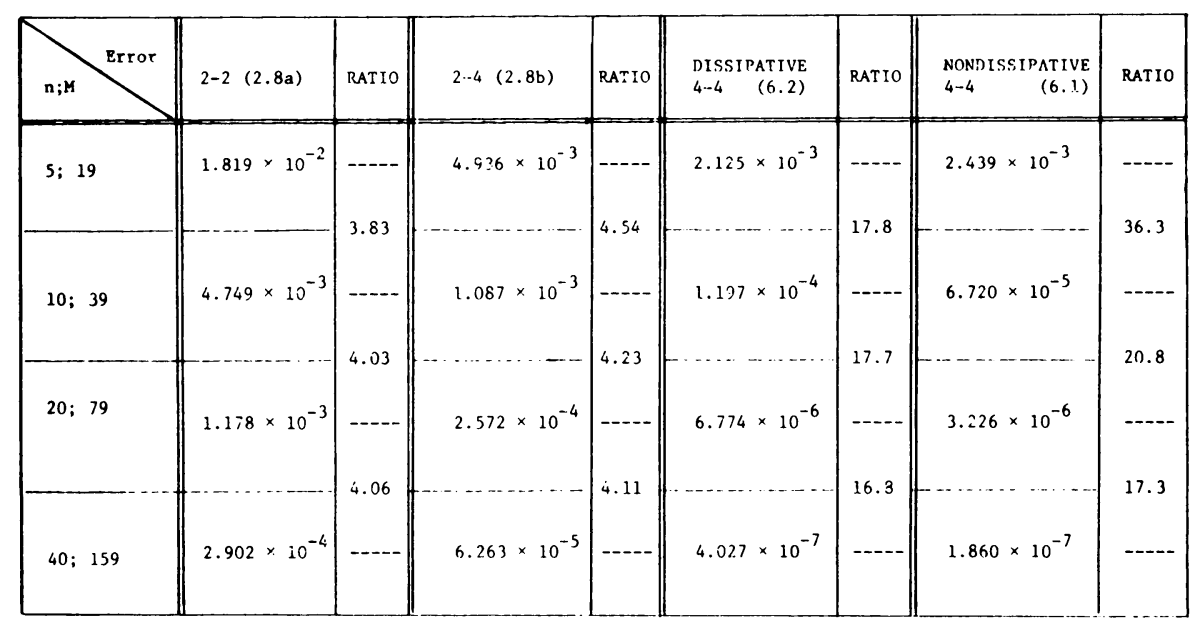

We turn now to numerical experiments with $d=2$ in (6.3). In this case the characteristic speed in (6.3) is always positive (the shock speed is 2 ) and we do not encounter nonlinear instabilities. In Table III, we present a mesh refinement chart for the 2-2 (2.8a), 2-4 (2.8b), dissipative 4-4 (6.2), and nondissipative 4-4 (6.1) schemes. $\Delta x$ and $\Delta t$ in this table are defined by

$$
\Delta x=2 \pi / M, \quad \frac{\Delta t}{\Delta x} \max _{v}|a(v)|=0.95
$$

The relative $L_{2}$-errors $(5.8)$ in this chart correspond to results at $t \approx 0.51$, at which the solution is still smooth. Table III clearly demonstrates the full fourth order accuracy of schemes (6.1) and (6.2). In Figures 4 and 5, we show numerical solutions of the dissipative 4-4 scheme (6.2), the 2-4 scheme (2.8b), and the one-step Lax-Wendroff scheme (6.1a), and compare them with the exact solution. $\Delta x$ and $\Delta t$ in these calculations are given by (6.4) with $M=39$. In Figure 4 , we show these solutions at $t=1.25(n=25)$, a short time after the formation of the shock. In Figure 5, we show the results of these computations at $t=5.02(n=100)$, at which time the shock has decayed considerably. The solution of the $2-4$ scheme $(2.8 \mathrm{~b})$ in Figure $4(b)$ is oscillatory to the point of being meaningless. Similar results are obtained by other nondissipative schemes.

Next, we describe numerical experiments with the following Riemann problem (shock tube) for the Eulerian equations of a polytropic gas:

$$
\begin{gathered}
w_{t}+f(w)_{x}=0, \quad w=\left(\begin{array}{c}
\rho \\
m \\
E
\end{array}\right), \quad f(w)=u w+\left(\begin{array}{l}
0 \\
P \\
P u
\end{array}\right), \\
P=(\gamma-1)\left(E-\frac{1}{2} \rho u^{2}\right), \\
w(x, 0)=\left\{\begin{array}{ll}
w_{L}, & x \leqslant 0, \\
w_{R}, & x>0,
\end{array} \quad w_{L}=\left(\begin{array}{l}
1 \\
0 . \\
2.5
\end{array}\right), \quad w_{R}=\left(\begin{array}{l}
0.125 \\
0 . \\
0.25
\end{array}\right) .\right.
\end{gathered}
$$




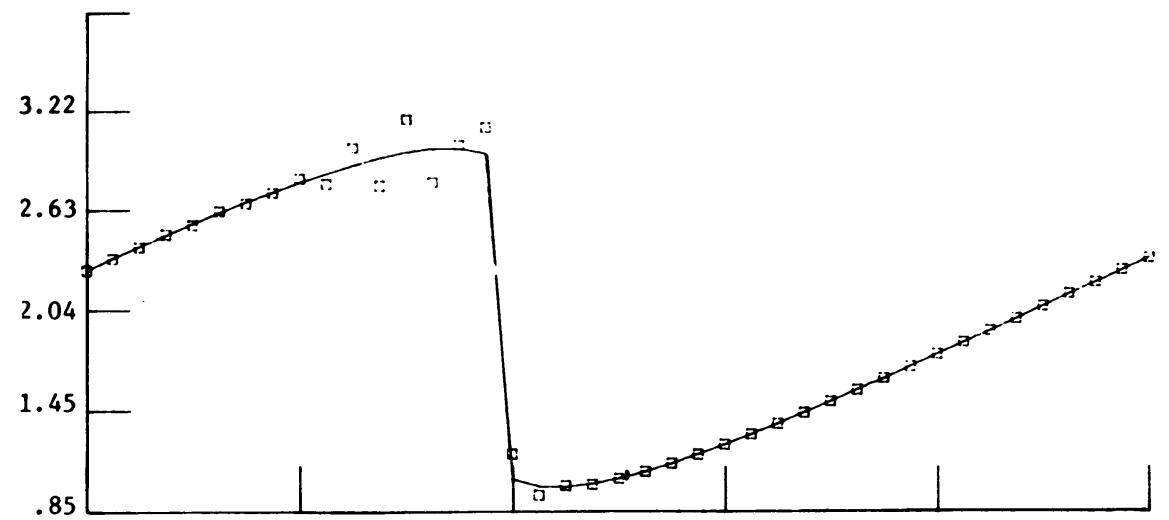

(a) Dissipative 4-4

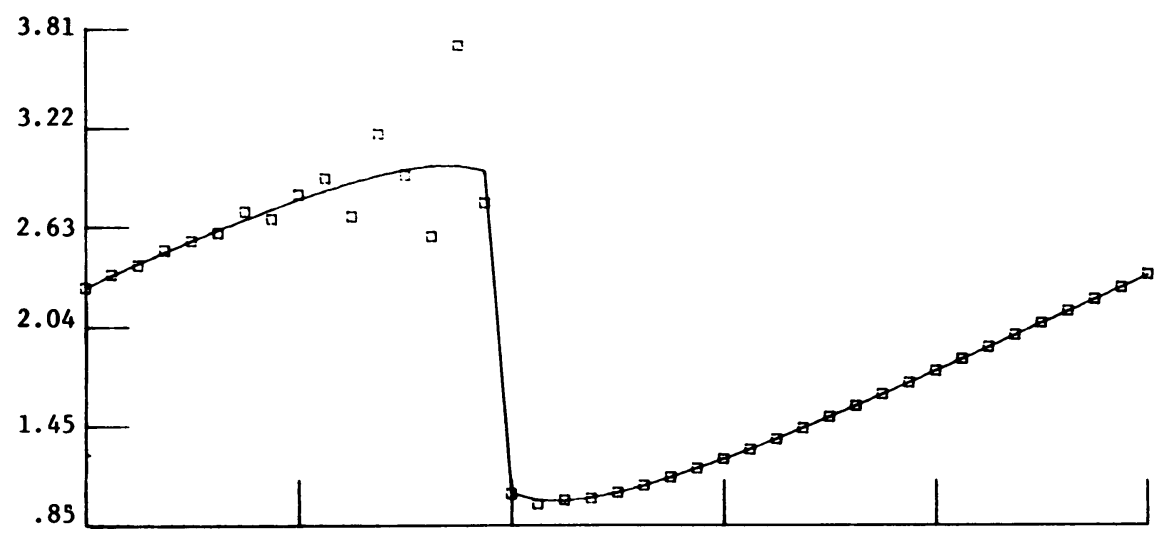

(b) Implicit 2-4

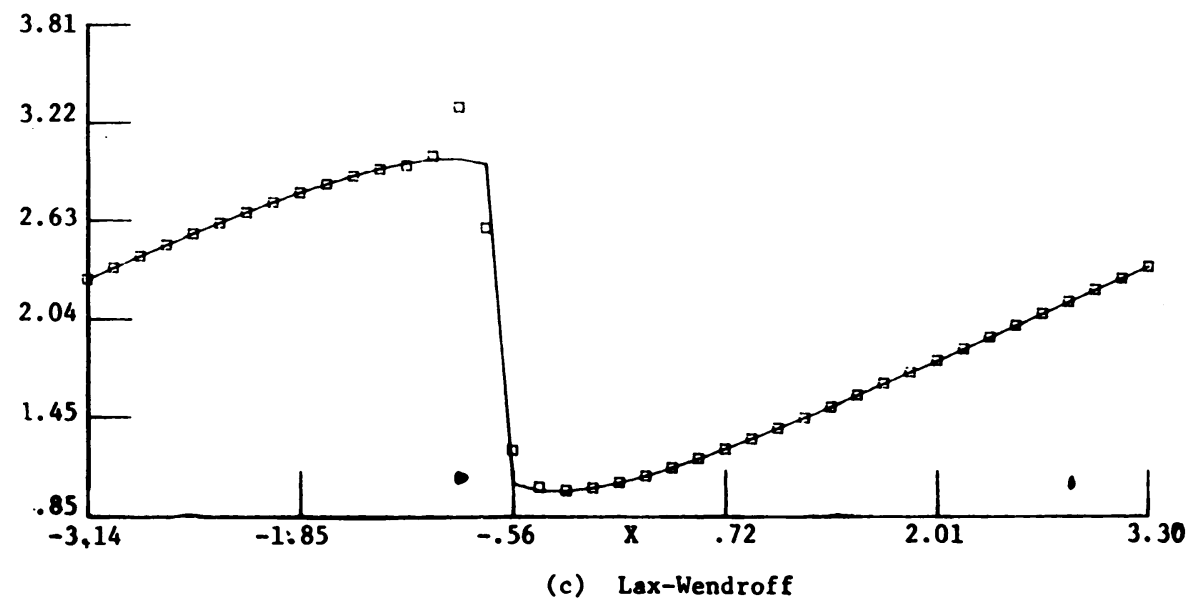

FigURE 4

Solutions to the nonlinear problem (6.3) at $t=1.25(n=25)$ 


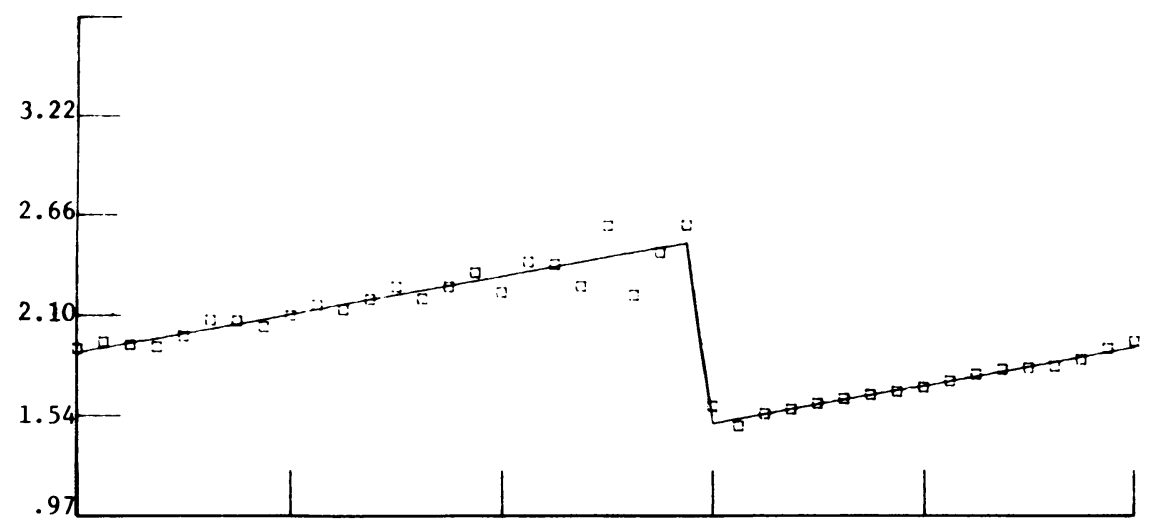

(a) Dissipative 4-4

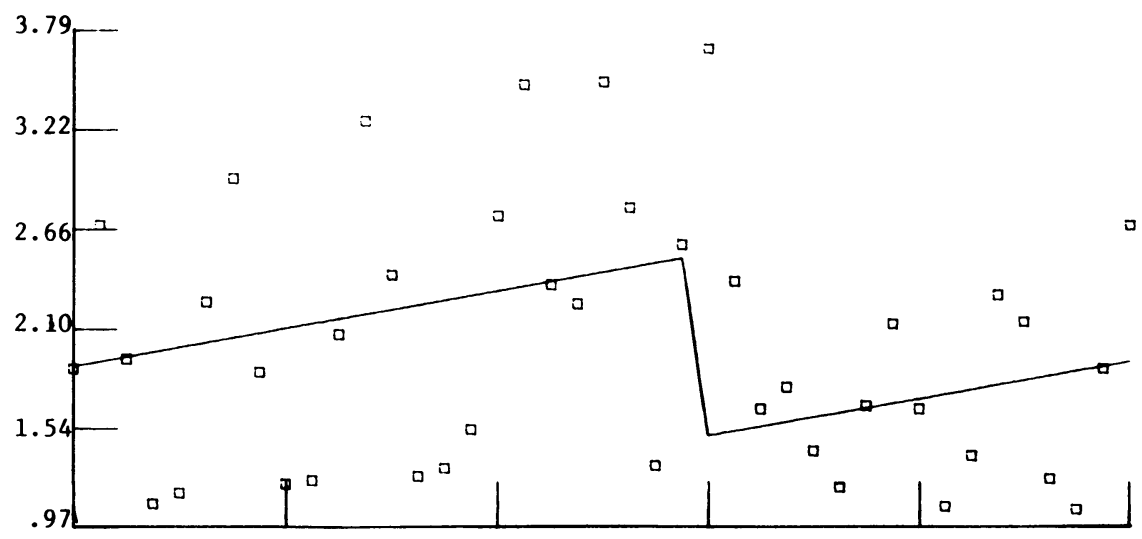

(b) Implicit 2-4

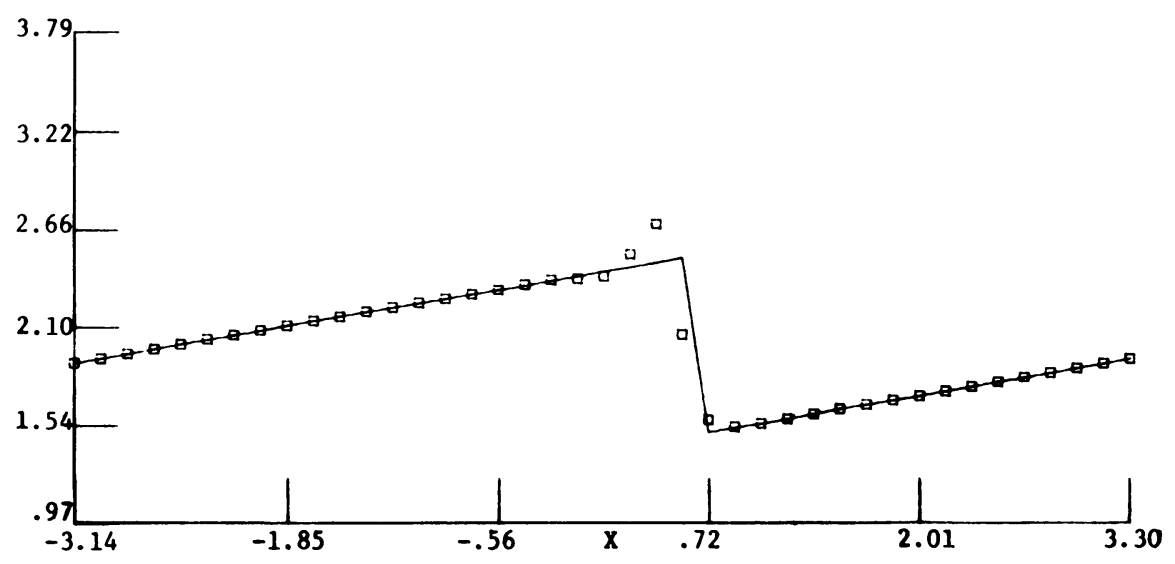

(c) Lax-Wendroff

Figure 5

Solutions to the nonlinear problem $(6.3)$ at $t=5.02(n=100)$ 

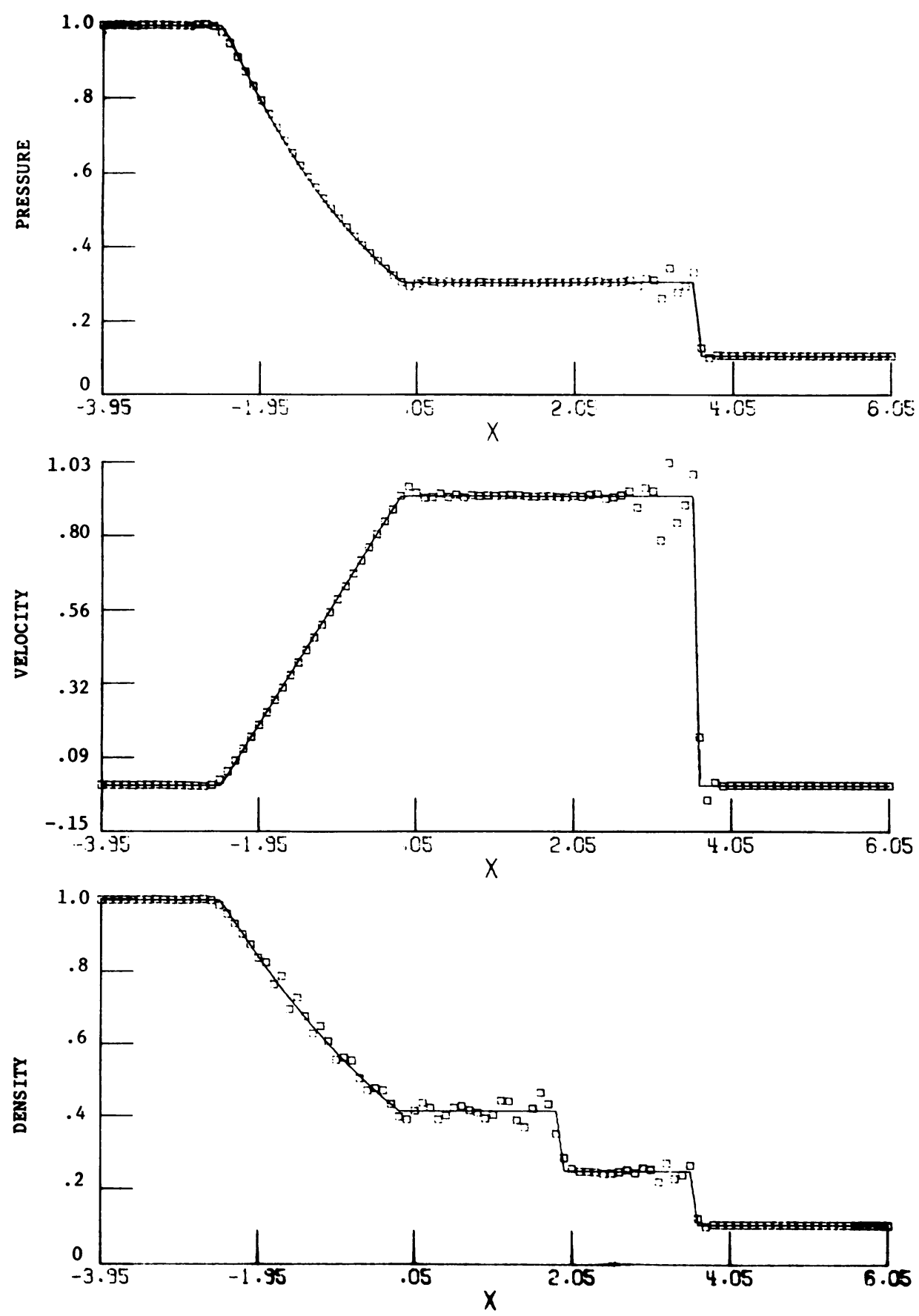

FIGURE 6

Dissipative 4-4 solution to the Riemann problem (6.5) 

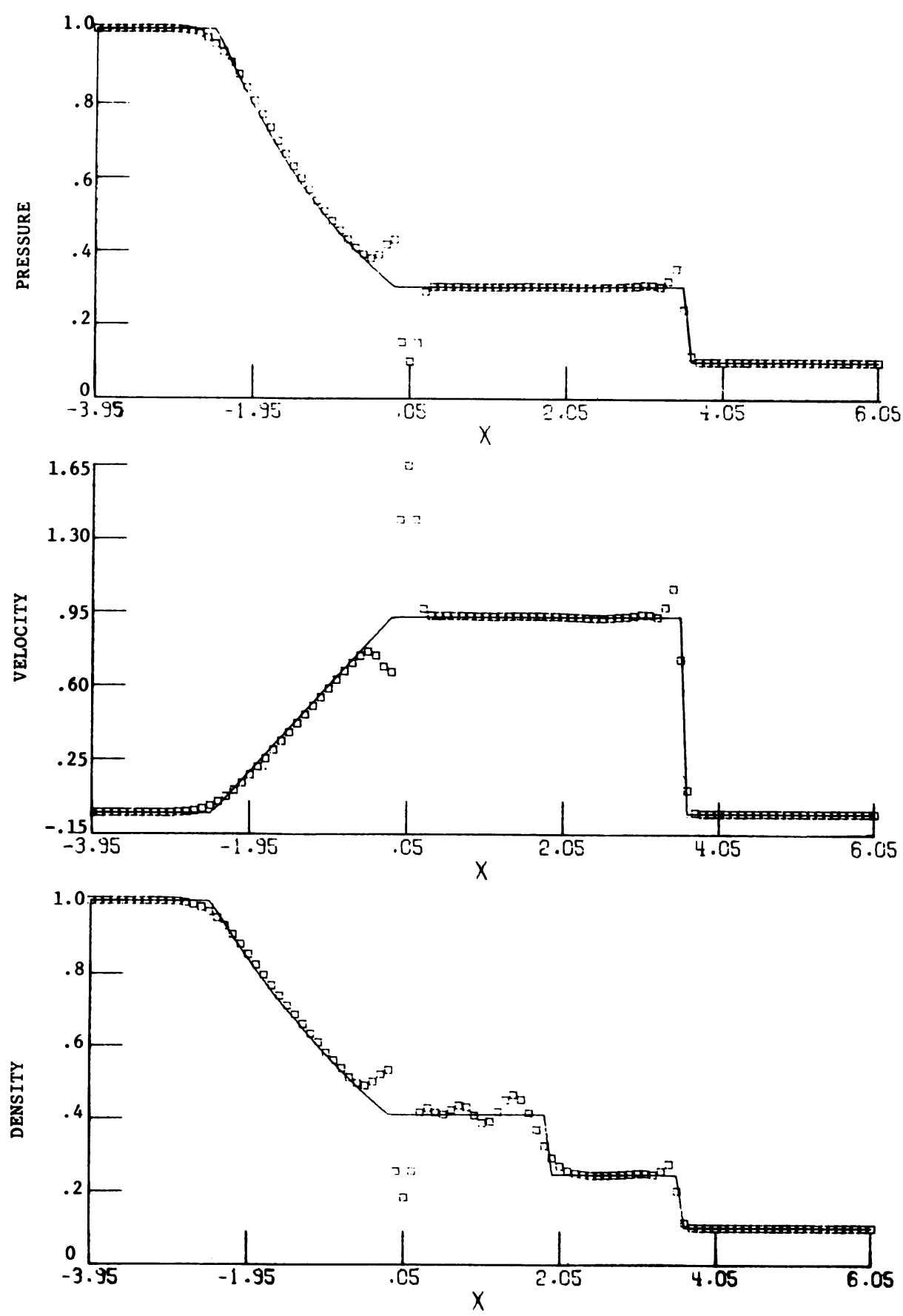

FIGURE 7

Lax-Wendroff solution to the Riemann problem (6.5) 
Here, $\rho, u, P$, and $E$ are the density, velocity, pressure and total energy respectively; $m=\rho u$ is the momentum, $\lambda=1.4$. The exact solution of this Riemann problem consists of a shock propagating to the right, followed by a contact discontinuity, and a left propagating rarefaction wave. This exact solution is shown in Figures 6 and 7 by a continuous line. A more detailed analysis of the structure of the solution to this particular Riemann problem, as well as comparison of its numerical solution by various finite difference schemes, are available in a review paper by Sod [14]. In Figure 6, we show the solution of the dissipative 4-4 scheme (6.2); for sake of comparison we show in Figure 7 the solution of the one-step Lax-Wendroff scheme (6.1a). Both figures show the solution at $t=2.05$ after 50 time-steps with $\Delta x=0.1 ; \Delta t^{(n)}$ is determined at the beginning of each time-step by

$$
\frac{\Delta t^{(n)}}{\Delta x} \max _{j}\left(\left|u_{j}^{n}\right|+c_{j}^{n}\right)=0.95
$$

where $c=(\gamma P / \rho)^{1 / 2}$ is the local sound speed.

We make the following observations and remarks concerning the numerical solutions of the discontinuous weak solutions in Figures 5-8:

(1) All numerical solutions in these figures produce correct propagation of discontinuities.

(2) Nondissipative schemes cannot handle shock problems. In fact numerical solutions of the 2-2, 2-4, and 4-4 nondissipative schemes of this paper become unstable (in the sense of developing negative density and pressure) in the solution of the Riemann problem (6.5).

(3) The 4-4 scheme produces sharp transition of discontinuities but with a long tail of post-shock oscillations.

We conclude that the dissipative 4-4 scheme has sufficient dissipation to remain stable in the strongly nonlinear case but not enough to damp post-shock oscillations in a satisfactory fashion. Some additional local dissipative mechanism is needed; such terms can be developed by hybridization techniques; see [5] and [2].

VII. Summary and Concluding Remarks. In this paper, the first in a series, we have presented a new implicit, compact, unconditionally stable, nondissipative, 4-4 accurate scheme for the numerical solution of one-dimensional systems of hyperbolic conservation laws. A more robust version of the basic scheme, which is dissipative and conditionally stable, was introduced for the solution of nonstiff strongly dynamic problems. These $4-4$ accurate schemes take only about $25 \%$ more computing time per time-step per mesh point than the 2-4 accurate Beam and Warming scheme (2.8b).

In this first paper, we have only explored some basic properties of the methodmuch more has to be done to make this 4-4 implicit scheme a viable computational tool. The immediate items to be investigated are: (1) stable and sufficiently accurate treatment of physically relevant boundary conditions, (2) better resolution of shock waves, and, most importantly, (3) efficient algorithms for multi-dimensional problems. 
Acknowledgement. We thank Alvin Bayliss for many helpful and stimulating discussions.

ICASE

NASA Langley Research Center

Hampton, Virginia 23665

1. S. Abarbankl, D. Gotrlieb \& E. Turkel, "Difference schemes with fourth order accuracy for hyperbolic equations," SIAM J. Appl. Math., v. 29, 1975, pp. 329-351.

2. R. M. BEAM \& R. F. WARMING, "An implicit finite difference algorithm for hyperbolic systems in conservation-law form," J. Comput. Phys., v. 22, 1976, pp. 87-110.

3. P. Davis \& P. RabInowitz, Methods of Numerical Integration, Academic Press, New York, 1974.

4. B. GustafsSon, "An alternating direction implicit method for solving the shallow water equations," J. Comput. Phys., v. 7, 1971, pp. 239-254.

5. A. HARTEN, "The artificial compression method for computation of shocks and contact discontinuities: III. Self-adjusting hybrid scheme," Math. Comp., v. 32, 1978, pp. 363-389.

6. A. HARTEN \& G. ZwAS, "Self-adjusting hybrid schemes for shock computations," J. Comput. Phys., v. 9, 1972, pp. 568-583.

7. A. Harten, J. M. Hyman \& P. D. Lax, "On finite difference approximations and entropy conditions for shocks," Comm. Pure Appl. Math., v. 29, 1976, pp. 297-322.

8. R. S. HIRSH \& D. H. RUDY, "The role of diagonal dominance and cell Reynolds number in implicit difference methods for fluid mechaics problems," J. Comput. Phys., v. 16, 1976, pp. 304-310.

9. E. IsAacson \& H. Keller, Analysis of Numerical Methods, Wiley, New York, 1966.

10. P. D. LAx, Hyperbolic Systems of Conservation Laws and the Mathematical Theory of Shock Waves, SIAM Regional Conference in Applied Math., 1973.

11. P. D. LaX \& B. Wendroff, "Systems of conservation laws," Comm. Pure Appl. Math., v. 23, 1960, pp. 217-237.

12. A. MNDA \& S. OshER, "A systematic approach for correcting nonlinear instabilities: The Lax-Wendroff scheme for scalar conservation laws," Numer. Math. (To appear.)

13. K. W. Morton, "Initial value problems by finite difference and other methods," The State of the Art in Numerical Analysis, Academic Press, New York, 1977, pp. 699-756.

14. G. A. SoD, "A survey of several finite difference methods for systems of nonlinear hyperbolic conservation laws," J. Comput. Phys., v. 27, 1978, pp. 1-31.

15. E. TuRkel, On the Practical Use of High Order Methods for Hyperbolic Problems, ICASE Report No. 78-19, NASA Langley Research Center, November 1978.

16. H. J. WIRZ, F. DeSchUTter \& A. TURI, "An implicit, compact, finite difference method to solve hyperbolic equations," Math. Comput. Simulation, v. 19, 1977, pp. 241-261.

17. A. HARTEN \& H. TAL-EZzR, On a Fourth Order Accurate Implicit Finite Difference Scheme for Hyperbolic Conservation Laws: I. Nonstiff Strongly Dynamic Problems, ICASE Report No. 79-1, NASA Langley Research Center, January 1979. 